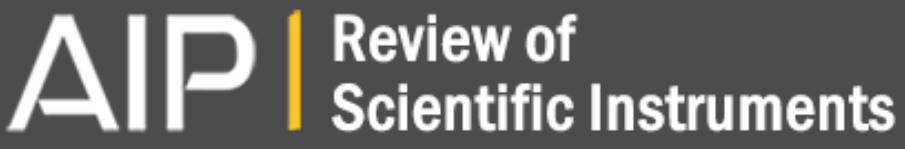

\section{Optical system for liquid level measurements}

D. Melchionni and M. Norgia

Citation: Review of Scientific Instruments 85, 075113 (2014); doi: 10.1063/1.4890437

View online: http://dx.doi.org/10.1063/1.4890437

View Table of Contents: http://scitation.aip.org/content/aip/journal/rsi/85/7?ver=pdfcov

Published by the AIP Publishing

Articles you may be interested in

Fiber-optic liquid level sensor based on coupling optical path length variation

Rev. Sci. Instrum. 83, 055006 (2012); 10.1063/1.4717725

Development of an optical three-dimensional laser tracker using dual modulated laser diodes and a signal detector

Rev. Sci. Instrum. 82, 035101 (2011); 10.1063/1.3549138

Asymmetric Fabry-Pérot fiber-optic pressure sensor for liquid-level measurement

Rev. Sci. Instrum. 80, 033104 (2009); 10.1063/1.3093808

Flexible lock-in detection system based on synchronized computer plug-in boards applied in sensitive gas spectroscopy

Rev. Sci. Instrum. 78, 113107 (2007); 10.1063/1.2813346

All-fiber-optic sensor for liquid level measurement

Rev. Sci. Instrum. 70, 2551 (1999); 10.1063/1.1149792

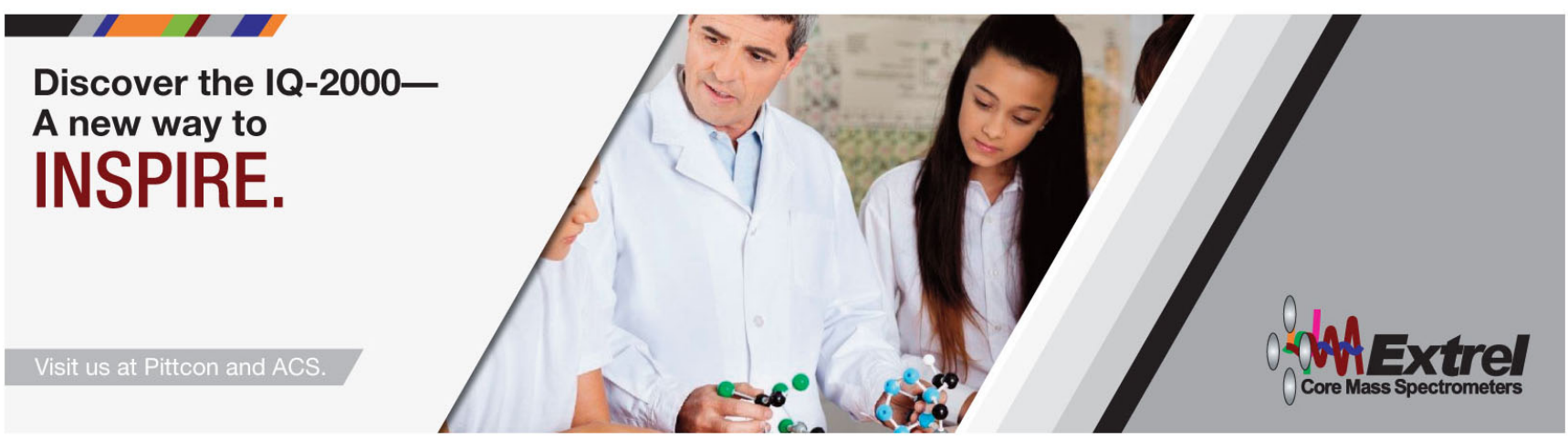




\title{
Optical system for liquid level measurements
}

\author{
D. Melchionni and M. Norgia \\ Dipartimento di Elettronica, Informazione e Bioingegneria, Politecnico di Milano, Milan, Italy
}

(Received 31 March 2014; accepted 6 July 2014; published online 22 July 2014)

This work describes the development of a novel laser instrument for non-contact liquid level measurements. The physical principle of operation is based on the self-mixing effect induced in the laser diode by modulating the laser wavelength. The frequency main tone of the resulting fringes is proportional to the absolute target distance. A high speed FPGA (Field Programmable Gate Array) electronic performs the data acquisition and elaboration. The high elaboration/acquisition speed improves the chance of acquiring good signal also on a moving liquid target. The optical characteristics of the liquid surface have been studied in order to identify the optimal optical configuration when the surface is flat as well as when the surface is rippled. The final low-cost instrument works in real time and is able to track a filling process with $2.5 \mathrm{~cm} / \mathrm{s}$ of speed, with resolution better than $1 \mathrm{~mm}$ for distances up to $50 \mathrm{~cm}$. @ 2014 AIP Publishing LLC. [http://dx.doi.org/10.1063/1.4890437]

\section{INTRODUCTION}

Liquid level measurements are fundamental in many industrial processes. Different methods can be found in the literature, but among them only a few perform contactless measurements. The actual trend is to replace mechanical and pressure-based measurement tools with systems that measure the distance to the fluid surface by a timing measurement. Ultrasonic and guided-wave radar are among the most versatile technologies available. ${ }^{1,2}$ However, the accuracy of these non-contact methods is low. Better performances are obtained with sensor based on fiber-optic. ${ }^{3,4}$ The fiber-optic liquid level sensing techniques generally rely on the interaction of the tip of the sensor with the surface of the liquid, or on transmitting light and receiving the reflected light from the surface. However, this technique has some drawbacks too: sensor exhibits large temperature sensitivity, every fiber-optic is limited to use with specific refractive index liquid and, most important, they can measure only if placed nearby the liquid surface. Therefore, none of these techniques is suitable for applications in which accuracy and distance are simultaneously required. In literature, interferometry has been used to achieve high accuracy, but only by positioning a floating retro-reflector on the liquid surface. ${ }^{5}$

The aim of this work is the development of a new optical instrument for liquid level measurement, able to reach a good tradeoff between performances and cost, directly measuring on the free liquid surface. The proposed solution is based on a coherent technique for laser diode (LD), known in literature as feedback interferometry or self-mixing. ${ }^{6,7}$ In recent years, the self-mixing technique was used for the design and construction of various types of measuring instruments, such as speed and displacement, ${ }^{8-11}$ absolute distance, ${ }^{12,13}$ flow, ${ }^{14-16}$ and vibrations measurement, ${ }^{17-19}$ also for biomedical applications. ${ }^{20}$ In this contribution, we propose a novel instrument for liquid level measurement, based on a self-mixing rangefinder, also analyzing the best optical configurations for working on liquid surfaces.

\section{SELF-MIXING INTERFEROMETRY}

The self-mixing effect is due to the reflection of a fraction of the emitted light into the laser cavity. This fraction of optical power modulates frequency and amplitude of LD. ${ }^{21}$ The measured power $P$ assumes a periodic form $F(\phi)$, function of the interferometric phase $\phi=2 k d$, where $k=2 \pi / d$, $d$ is the absolute target distance and $\lambda$ is the LD wavelength. $F$ function shape depends on the amount of back injected power quantified by the feedback parameter $C .{ }^{6}$

As illustrated in Fig. 1, by increasing the $C$ parameter from the very low back-injection to low back-injection or moderate one causes the fringes to change shape from sinusoidal to sawtooth like.

When the target is stationary at distance $d$, fringes can be induced by modulating the laser current. In fact, current modulation modifies the laser wavelength $\lambda$ due to thermal effects that change laser cavity dimension. This phenomenon induces a phase-shift of the back-injected light, which can be calculated by differentiating phase $\phi$ with respect to the current, $I$ :

$$
\frac{\partial \phi}{\partial I}=\left(-4 \pi \frac{s}{\lambda^{2}}\right) \cdot\left(\frac{\partial \lambda}{\partial I}\right),
$$

where the factor $(\partial \lambda / \partial I)$ is a specific parameter of the $L D .^{22}$

Fig. 2 qualitatively describes the signal detected from the photodiode when the current is modulated by a triangular wave. The obtained signal, due to modulation (reported on the left side), is shown on the right side. It is made of two main contributes: the triangular power modulation, due to directly changes in current, and superimposed fringes, due to interferometric phase change.

When the target is at rest, the fringes main tone frequency $f_{\text {tone }}$ is function of the distance $d,{ }^{13}$ and it is possible to keep a constant instrument sensitivity $S$ assuming that the slope of the modulation signal and the factor $(\partial \lambda / \partial I)$ are constant. The 


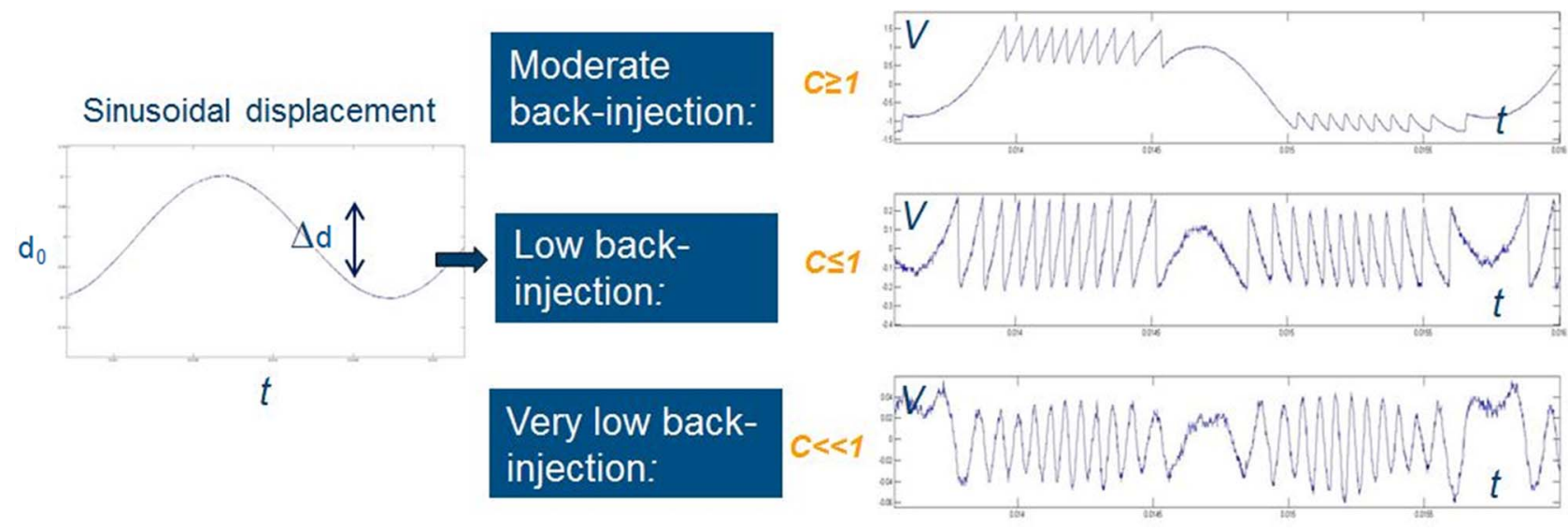

FIG. 1. Signal obtained over a target with sinusoidal displacement for different $\mathrm{C}$ values.

sensitivity $S$ is ${ }^{13}$

$$
d=-\frac{\lambda^{2}}{2 \cdot\left(\frac{\partial \lambda}{\partial I}\right) \cdot\left(\frac{\partial I}{\partial t}\right)} f_{\text {tone }}=S \cdot f_{\text {tone }} .
$$

\section{MEASUREMENT METHOD}

The proposed instrument aims to guarantee a measure of the liquid level even though the back reflected power quantity varies rapidly and chaotically due to the surface optical nature. Thus, the method chosen for the distance estimation has to extract the information in heterogeneous conditions of signal. The system needs three main elements to work properly: analog filtering, analysis in the frequency domain, and high acquisition speed.

As shown in Fig. 2, the analog signal amplified by the transimpedance is composed by the sum of the direct power modulation and the interferometric fringes. At this point, the analog signal is filtered and the LD differential efficiency contribution is partially subtracted. While calibrating the system, the LD differential efficiency must be compensated, in order to cancel the triangular wave amplitude modulation. It is not mandatory to exactly remove the triangular wave, but the operation must be repeated when changing the $\mathrm{LD}$, because each LD exhibits different parameters. A band pass filter allows removing noise, disturbances, and low frequency spurious signals. Such signal conditioning returns the signal shown in Fig. 3, showing the interferometric fringes also in the case of not-optimal optical conditions (low back-injection).

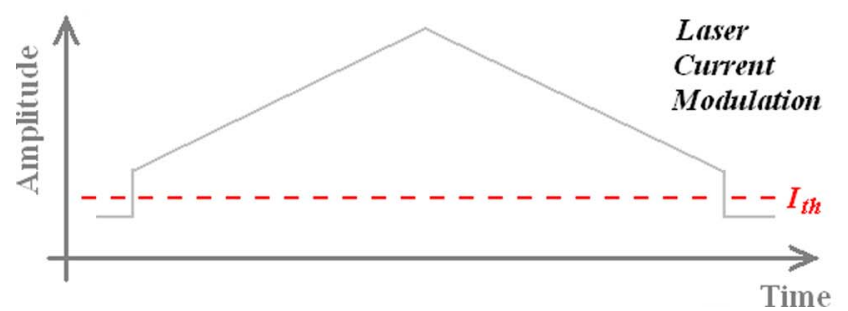

The proposed system estimates the distance $d$ from this signal, by evaluating the main frequency tone as previously expressed. This technique, deeply studied in a previous work, ${ }^{13}$ is more effective than the counter of fringes number in the time domain because it works also for very low $C$ value. Thus, it is applicable in every optical condition and guarantees a valid distance value even if the signal is affected by noise and disturbs.

The final point is about the acquisition rate. The chance to evaluate the distance in a short time period makes the measurement independent from the target movement. Indeed, with a short modulation period, the single measurement behaves as instantaneous with respect to the target movements, and the frequency induced by the modulation is much higher than the Doppler frequency induced by the target movement. For example, if we assume that the liquid level increases with a $4 \mathrm{~cm} / \mathrm{s}$ speed, a $40 \mathrm{kHz}$ modulation frequency is enough to limit the error due to the target movement. The effective movement during the semi-period, indeed, is limited to only $200 \mathrm{~nm}$. Thus, reducing the time of a single measure increases the accuracy and also the possibility of obtaining a valid signal on a variable surface. Moreover, it allows to average over a greater number of acquisitions $(N)$ and gaining a $\sqrt{ } N$ factor on the measurement standard deviation. However, it is not useful to increase $N$ indefinitely because of the laser thermal instability. As evident in the final measurements, the repeatability is limited because the parameters in formula (2) are not stable in time.

The choice of the frequency modulation, $40 \mathrm{kHz}$, is limited by the frequency response of the laser diode modulation

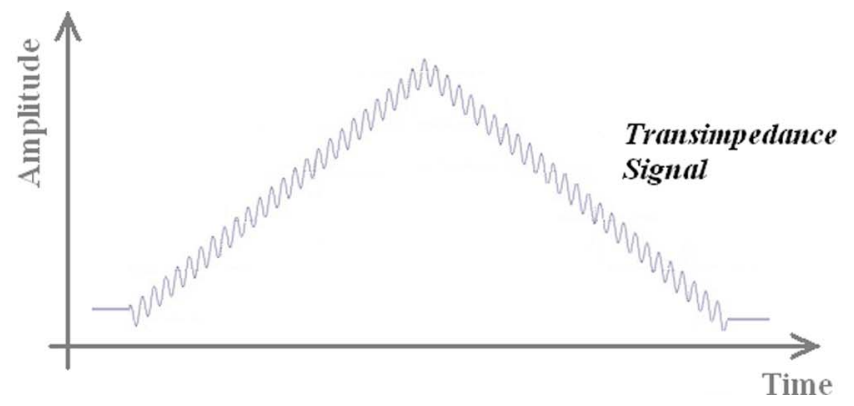

FIG. 2. Qualitative fringes generated by a triangular current modulation. 


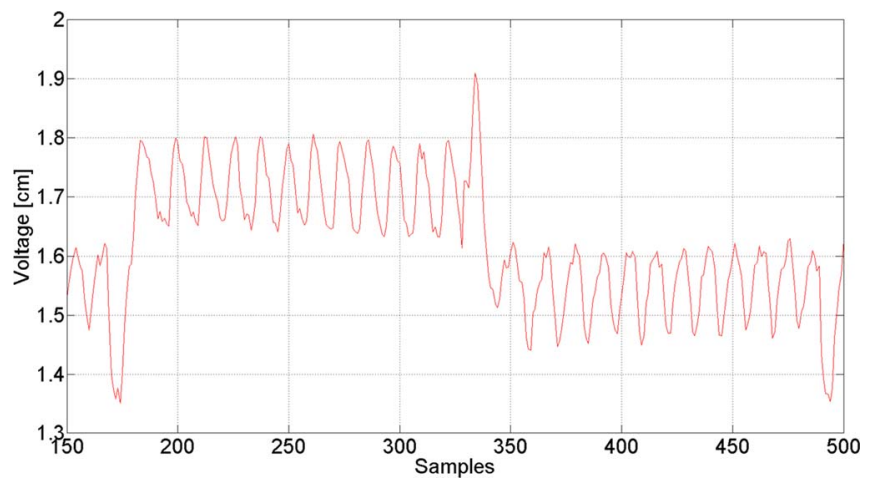

FIG. 3. Acquired fringes after the subtractor and pass-band filtering, for low back injection.

efficiency $(\partial \lambda / \partial I)$. For the used LD it exhibits a pole at about $120 \mathrm{kHz} .{ }^{22}$ The modulation amplitude depends instead on the desired fringes frequency and the operation distance. The minimum number of fringes necessary to apply successfully the FFT algorithm (about 5 fringes) determines the low limit of frequency modulation. On the other side, the maximum is fixed by the sample frequency $f_{s}$ which imposes a maximum fringes frequency $f_{\text {tone }}$ of about $f_{s} / 2$. However, the fringes frequency depends on frequency modulation as well as distance range, so the final frequency range is determined by both of these factors(2).

\section{HARDWARE SETUP}

The instrument design is divided in four parts and each part is subdivided in blocks as shown in Fig. 4. The main parts are: the optical system, the analog circuit, the digital circuits, and the PC acquisition and elaboration. In order to describe the basic function of each part it is useful to follow the measurement process from the laser beam generation to the final results. Furthermore, it is necessary to underline that this novel hardware architecture allows a massive improvement of the measurement speed compared with other instruments based on self-mixing technique already developed in previous works. $^{13}$

First, as shown in Fig. 4 (left) the optical part is made by a LD (two LDs were tested: model HL7851G, $785 \mathrm{~nm}$ and model DL7140, $785 \mathrm{~nm}$ ) and a plastic lens which allows to change the focusing conditions. The next paragraph comments the implementation of the optical setup. In the next part, the analog circuitry elaborates the signals before the acquisition. A transimpedance amplifier reads the current signal and partially subtracts the triangular wave due to power modulation; in fact, laser current variations change the optical power because of LD differential efficiency. A second stage filters the signal and amplifies it to fit the input range of the differential A-D converter. The single ended signal is transformed in double ended for minimizing the disturbances due to the digital circuitry.

The digital part generates the modulation square wave, manages the ADC (Analog to Digital Coverter), and transmits the acquired data to a PC. The signal is sampled by the ADC (Texas Instruments, THS1230, 12 bit) working at $12.5 \mathrm{MHz}$. Data transmission is performed by a FTDI (Future Technology Devices International) driver, a parallel FIFO (First-In First-Out) to USB (Universal Serial Bus) serial transmission interface. The instrument performs about 500 acquisitions/s, each acquisition contains three measurements, and the single measurement is made in about $25 \mu \mathrm{s}$.

\section{IMPLEMENTED DATA PROCESSING}

A PC performs the real time signal processing through a LabVIEW program. It reads 1000 samples from the USB every $2 \mathrm{~ms}$, and elaborates the signal in the frequency domain. The elaboration core is the algorithm for estimating the main tone frequency. For this particular application of measurement over water, a very high measurement accuracy is

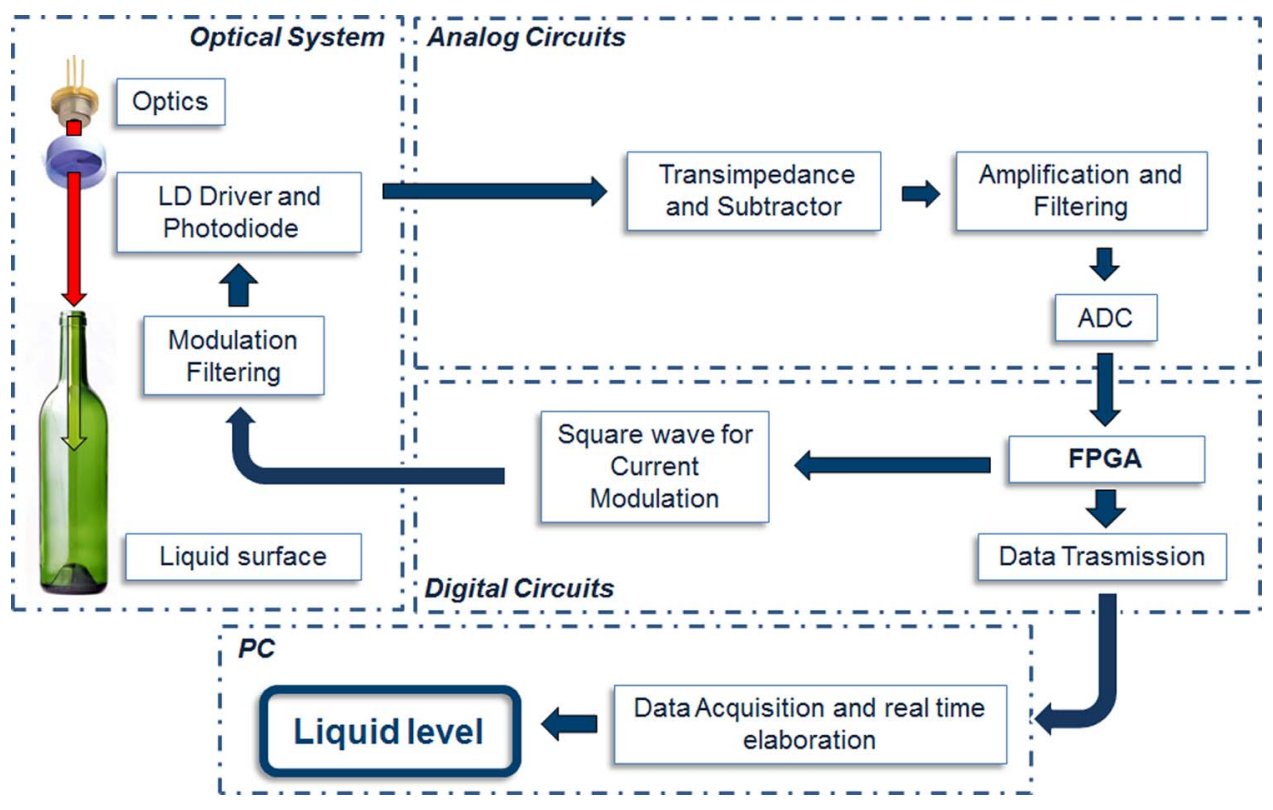

FIG. 4. Block diagram of the self-mixing liquid level instrument. 

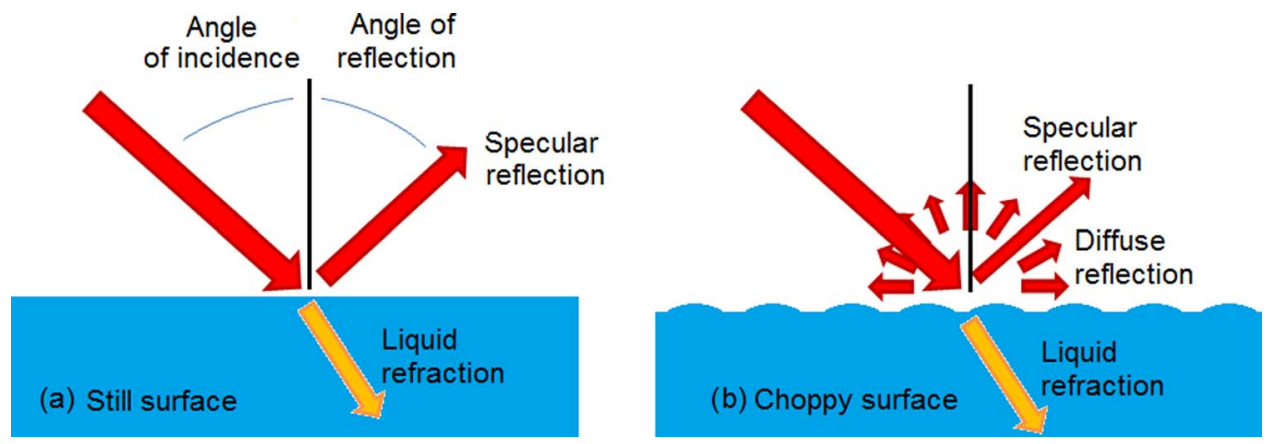

FIG. 5. Liquid surface optical behavior: (a) Still surface. (b) Rippled surface.

not really required, but the main goal is to obtain a reliable measurement also in presence of strong changes in the signal amplitude. Thus, the elaboration is first focused on verifying the quality of the acquired signal, and considering it only if high enough. After that, the distance is evaluated as in Ref. 13. The signal frequency is estimated after gating the signal, avoiding the beginning and the end of each modulation period that exhibit distortions. After some trying, the correct gating is obtained by minimizing the standard deviation of the distance measurement. In the second step, the frequency and the amplitude of the interferometric signal are calculated for the main tone, in correspondence to each rise and fall of modulation. At this point, a control is applied to each semi period to check the measurement validity. The control is on both the tone amplitude and frequency. The second control allows eliminating measurements when the tone amplitude is under a fixed threshold. In this case, in fact, the values extracted are not reliable due to low signal to noise ratio, and the measurement is discarded. The control on the frequency is useful to avoid signals from spurious reflections. It is implemented when the variation of liquid level is physically limited between two known values. In this case signals with "out-of-range frequencies" caused by spurious reflections are promptly suppressed. In addition, a monitoring has been applied also on the standard deviation of the three distance values calculated from the three measurement periods acquired. Each measurement is the average between rising and falling of the triangular wave. As explained in Ref. 13 this average reduces the effect of eventually target movements. Considering that the acquisition time has a duration of about $25 \mu \mathrm{s}$, it is not physically possible that the liquid surface moves too much. Thus, if high standard deviation is detected between the three consecutive measurements, the particular acquisition is not reliable.

When all the conditions are satisfied, the distance is calculated by applying (2) and averaging over the three measurements.

\section{OPTICAL SYSTEM FOR DIFFERENT SURFACE CONDITIONS}

Understanding the optical behavior of liquid surface is fundamental for this project. It is necessary to describe how the liquid surface reflects the laser beam in different condi- tions, in order to optimize the optics for measuring the liquid level.

Measuring the liquid level with optical technique is difficult because of the variability of the surface, and because the reflection is only due to the change of refractive index. In order to simplify the analysis we consider two cases: still and rippled water surface, such as during a filling process.

In the first scenario, the liquid is motionless and the surface is flat. Thus, it is reasonable to assume that its optical behavior is similar to a mirror, which reflects the optical power in a coherent manner. Despite a perfect mirror, just a portion of optical power is back reflected, the other one is refracted (Fig. 5(a)). On the other side, if a liquid is shaken at high frequencies, it could become similar to a target with a rough surface. In this case, a portion of the laser beam is no more reflected, but it can be scattered in different directions, just like a diffusive target (Fig. 5(b)).

A good self-mixing signal is obtained when a fraction between $10^{-6}$ and $10^{-4}$ of power is back injected in the laser cavity. However, $C$ value changes rapidly due to variations of liquid surface, so the system needs to work properly for different back injection values. This is guaranteed by the robust signal processing technique. In addition, the optical system maximizes $C$ value when it is low (choppy surface), and, on the other hand, avoids laser instability when the optical power back injected is high (still surface) ${ }^{23}$ For keeping the laser in the most suitable condition, it is possible to act on the position of the collimating lens. This means changing the focusing condition in order to optimize the probability of acquiring valid measurements.

The two scenarios are optically different. The first one can induce laser instability when the reflected beam is perfectly redirected in the cavity, but it is extremely sensitive to deviation from the perpendicularity condition between laser and liquid surface. The reason is the unidirectional reflection of the surface. In the second scenario, the optical power is spread due to the diffusion of the choppy surface. Therefore, there is always a possibility of reading signal, even if not perpendicular to water surface, but the amplitude could be very low.

These assumptions are the guidance for the optical configuration adopted and experimentally proved. In case of flat surface, the best working is obtained when positioning the focus before the surface, as shown in Fig. 6(a). In this way the angular acceptance is deeply increased, and there is no need 


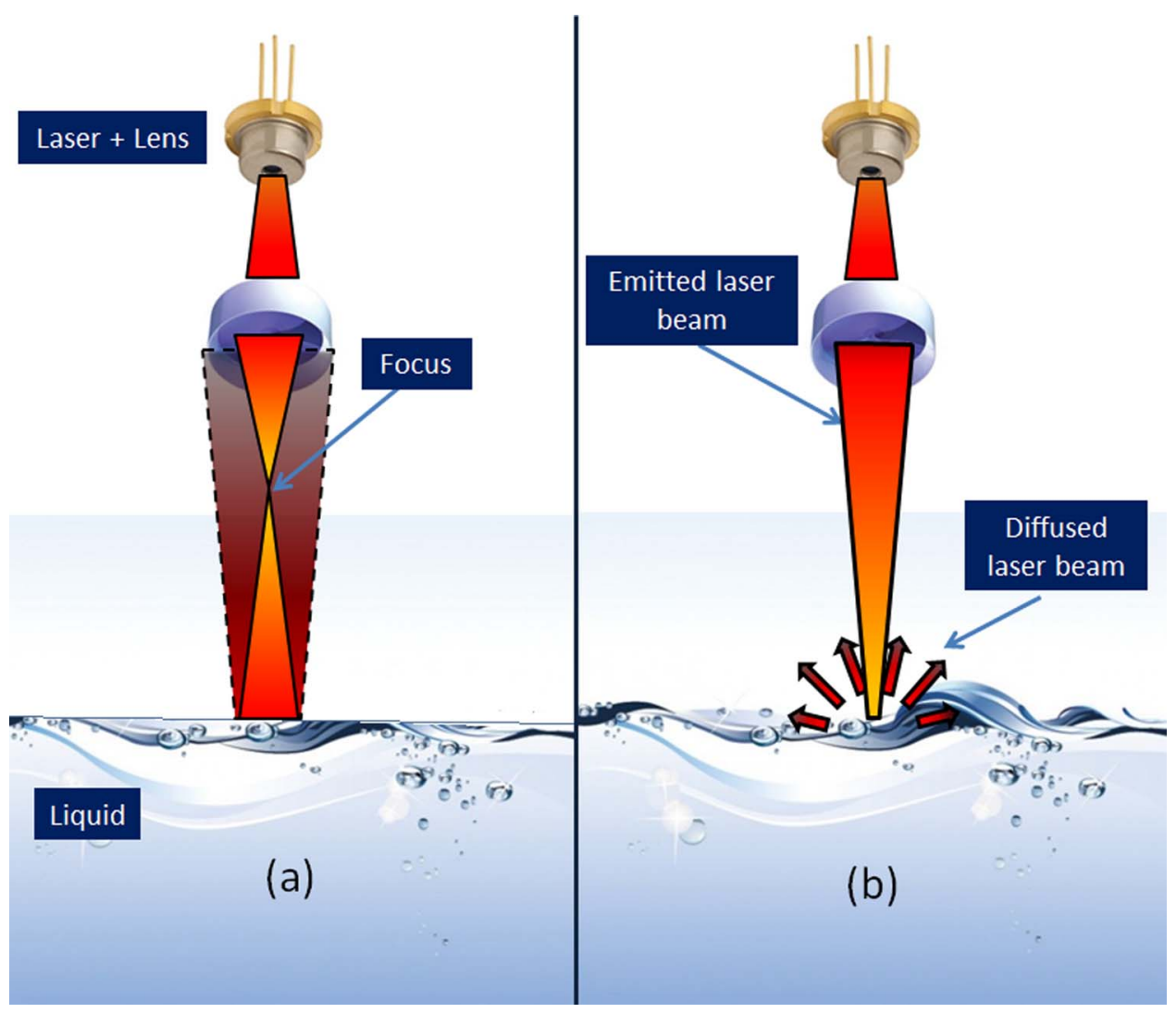

FIG. 6. Optical configuration: (a) Focus before the surface. (b) Focus shortly after the surface.

for perfect verticality of the laser beam. Moreover, the selfmixing effect is strongly attenuated, due to the partialization of the laser beam back-reflected into the laser cavity, and there is no more risk of too high back-injection level.

When the liquid surface is rippled at high frequency, such as during a filling process, the optical situation changes drastically, and the water tends to behave like a solid diffuser: the diffusion naturally spread the power, therefore the beam needs to be focused on the target to increase the back reflected signal (just like a solid target). Fig. 6(b) shows this second situation.

In conclusion, a trade-off between the intensity of selfmixing signal and the angle of view exists and it considerably depends on the liquid state of agitation. However, the working condition can be optimized by properly changing the laser focusing.

The laser verticality remains very important in both cases. Indeed, the system depicted in Fig. 6(a) can work for a misalignment of a few degrees, depending on the desired distance range to be measured. The condition of Fig. 6(a) needs a good verticality too, because the maximum direction of back-diffusion is always the specular reflection. Experimentally we found about 3 degrees of vertical tolerance for $30 \mathrm{~cm}$ of measurement range. It is worth to note that the signal back-reflected is due only to the jump of refractive index between air and water, and it is independent of the liquid purity. The following experimental results are shown for pure water, but we had very similar results also for other liquid, such as drinks, vine or beer. A significant difference appears only in the presence of strong scatterers placed inside the liquid: in that case a laser focusing like Fig. 6(b) could induce signal also from the scatterers inside the liquid, therefore spoiling the liquid level measurement. On the contrary, an ink does not influence the level measurement, because it attenuates the signal from inside the liquid, but not the reflection due to the refraction index change.

The laser coherence length intrinsically limits the measurement range of this kind of instruments, for the used laser diode it is equal to about $10 \mathrm{~m} .{ }^{24}$ However, for the realized setup, the main limit is the optics: with a simple collimating lens, the number of valid acquisitions is acceptable in a range from about $5 \mathrm{~cm}$ to $70 \mathrm{~cm}$, useful for liquid-level industrial application. If needed, a beam expander could be used to extend the distance range.

\section{EXPERIMENTAL RESULTS}

The realized self-mixing instrument for liquid level measurement was tested in different conditions. In the following, some tests are presented in order to show its performances for different optical focusing and liquid conditions. As previously explained, the limiting factor is the presence of a valid signal rather than the accuracy of the self-mixing technique. For comparison, the performances of the instrument on a white paper target are also shown.

We tried two infrared lasers sources: model HL7851G, $785 \mathrm{~nm}$ and model DL7140, $785 \mathrm{~nm}$. Both of them guarantee a good interferometric signal, but HL7851G has a $(\partial \lambda / \partial I)$ greater than DL7140, so, comparing them at the same 


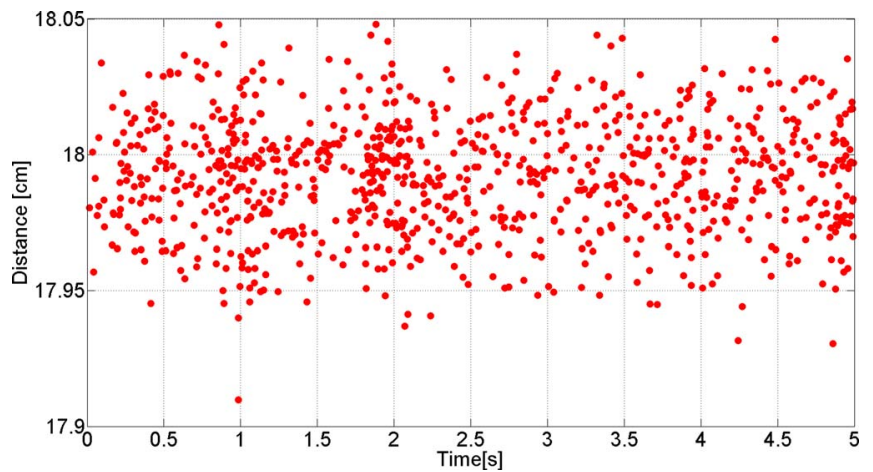

FIG. 7. Distance measured on a white paper, with a laser focus shortly after the target.

distance, it generates more fringes. The reported results are for the model HL7851G, but the performances obtained with the other model are comparable. The distance range of the instruments is set from $5 \mathrm{~cm}$ to $55 \mathrm{~cm}$, corresponding to about $337,5 \mathrm{kHz}$ and $3,7 \mathrm{MHz}$ of fringes frequency. The current polarization is $70 \mathrm{~mA}$, with current peak to peak modulation $\Delta I$ $=10 \mathrm{~mA}$, frequency modulation $f_{m}=40 \mathrm{kHz}$, and sample frequency $f_{s a}=12.5 \mathrm{MSa} / \mathrm{s}$. The instrument sensitivity experimentally calibrated is $S \cong 14.8 \mathrm{~cm} / \mathrm{MHz}$. In the following, some measurements at a distance of about $18 \mathrm{~cm}$ are shown, first on white paper, then over pure water. Every data set was recorded during a five seconds of acquisition. Each valid measurement is obtained applying the three controls with an amplitude threshold slightly greater than the noise and a deviation standard filter set to $1 \mathrm{~cm}$ on the acquired signal.

In Figs. 7 and 8 are shown the measurements obtained on a white paper target, with the focus respectively shortly after the target and before. In this case, there is no evidence supporting one or the other optical setup: the measurement technique is robust against signal amplitude, and on a solid target it exhibits a good working. In both cases, the measurements have a standard deviation under $0.16 \mathrm{~mm}$, and about 200 valid values per second.

The optical configuration is instead crucial to increase the number of measurements, when the target is a flat liquid surface. In this case, the focus before the target augments the probability with respect to the other configuration, as it is evident in Figs. 9 and 10. For comparison, the measurements

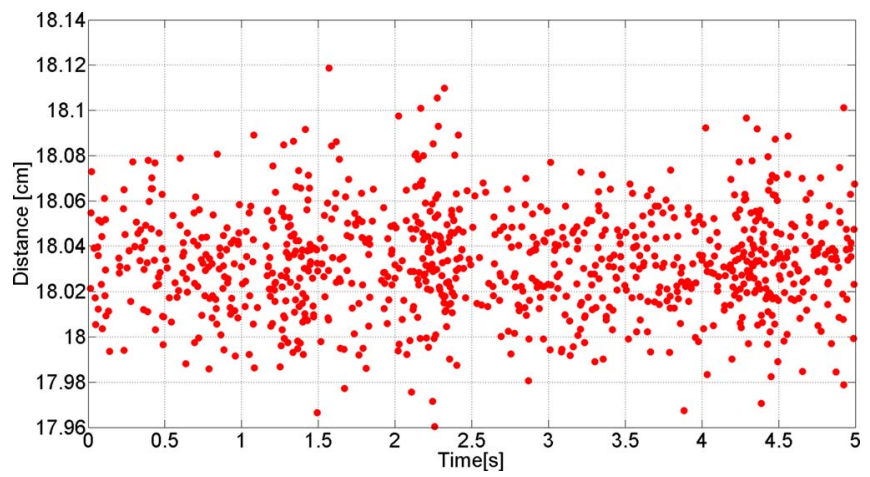

FIG. 8. Distance measured on a white paper, with a laser focus before the target.

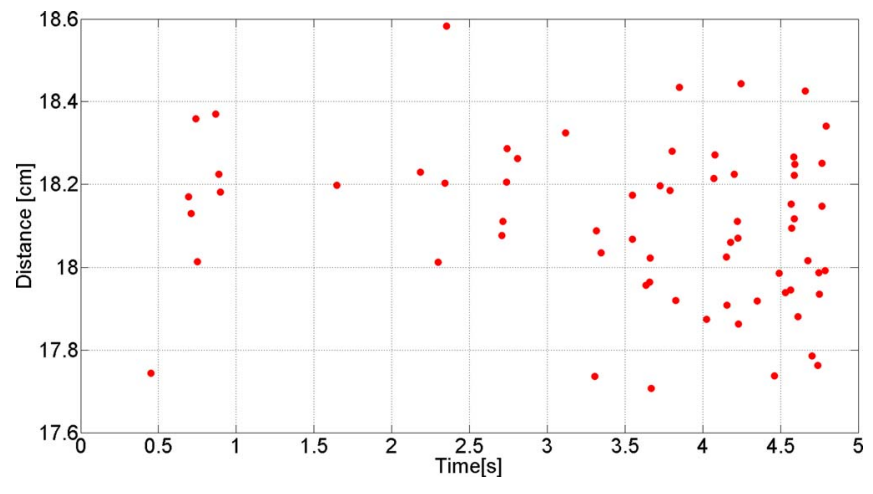

FIG. 9. Distance and standard deviation on liquid still surface. Focus shortly after the target.

are always taken at a distance of about $18 \mathrm{~cm}$. In Fig. 9 the focus is shortly after the water, in Fig. 10 it is before. It is evident that the probability of a good measurement is more than doubled in the second condition.

Figures 11 and 12, instead, show the measurements on a rippled liquid surface. In this case, it is extremely clear that the best configuration is the one with the focus shortly after the target surface (Fig. 11) both for number of valid acquisition and standard deviation. An ultrasonic washer, added to a manual low frequency agitation, induced the liquid movement. This situation simulates the ripples induced by an industrial filling process.

The measurements results at a fixed distance are confirmed also during the monitoring of a filling process. Fig. 13 reports three different tests of an industrial filling process: each point is a measurement over thresholds. The liquid level was increased with a filling velocity of about $2.5 \mathrm{~cm} / \mathrm{s}$. The three different fillings are described correctly by the instrument measurements. During the process the surface is shocked by the falling liquid itself, therefore the best is reached with the focus on the container bottom, after the target surface.

Fig. 14 shows the same measurement made with the laser focus placed before the target. As shown before, the laser sensor takes many good measurements at the beginning, when the water is firm. No measurement point is taken during the filling process, and a few points are taken at the end, when the water is still moving slowly without high-frequency agitation.

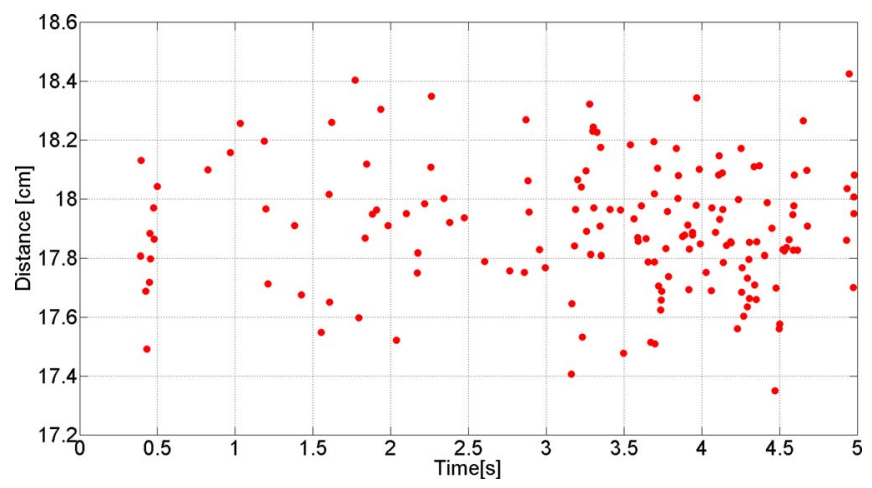

FIG. 10. Distance and standard deviation on liquid still surface. Focus before the target. 


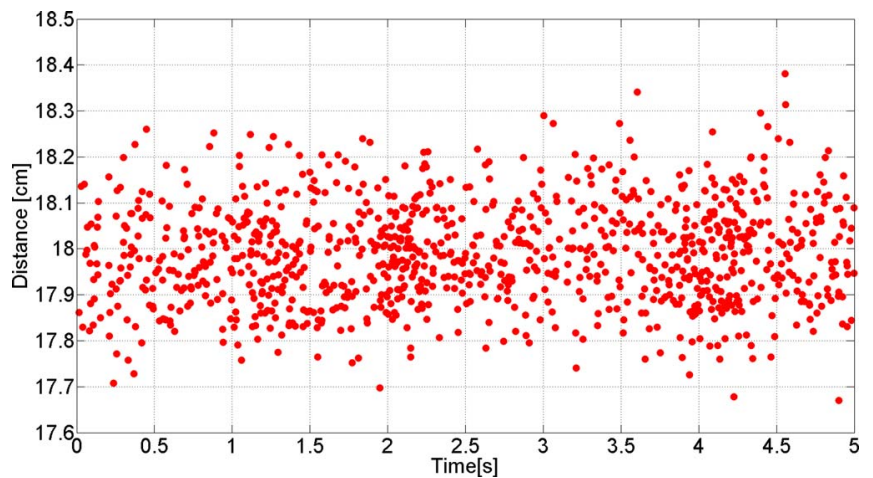

FIG. 11. Distance and standard deviation on liquid rippled surface. Focus shortly after the target.

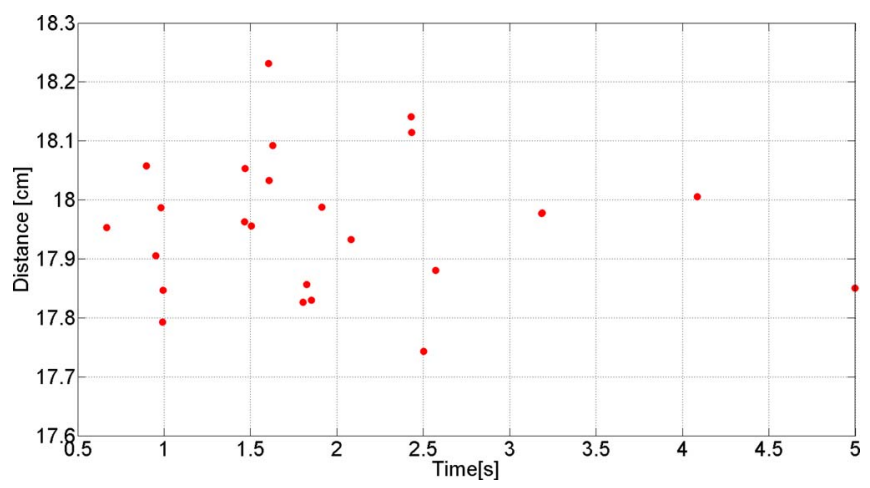

FIG. 12. Distance and standard deviation on liquid rippled surface. Focus before the target.

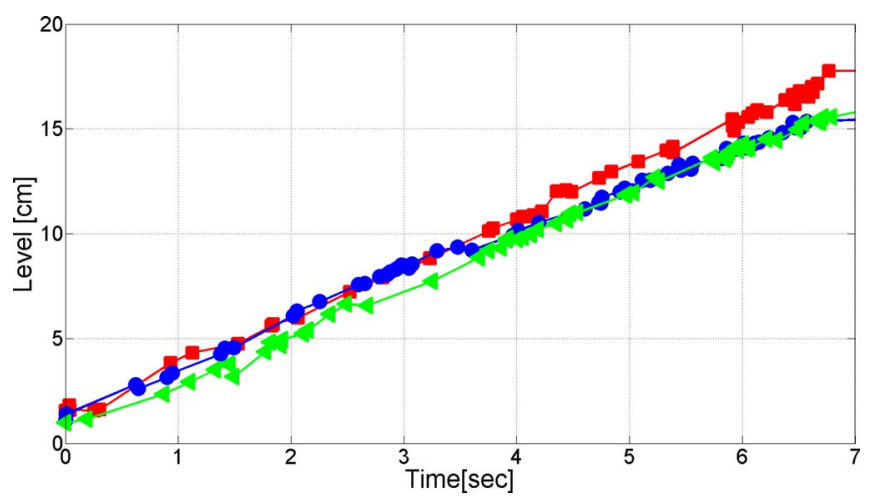

FIG. 13. Liquid level measurements during an industrial filling process. Focus shortly after the target. Average distance of the surface was around $40 \mathrm{~cm}$.

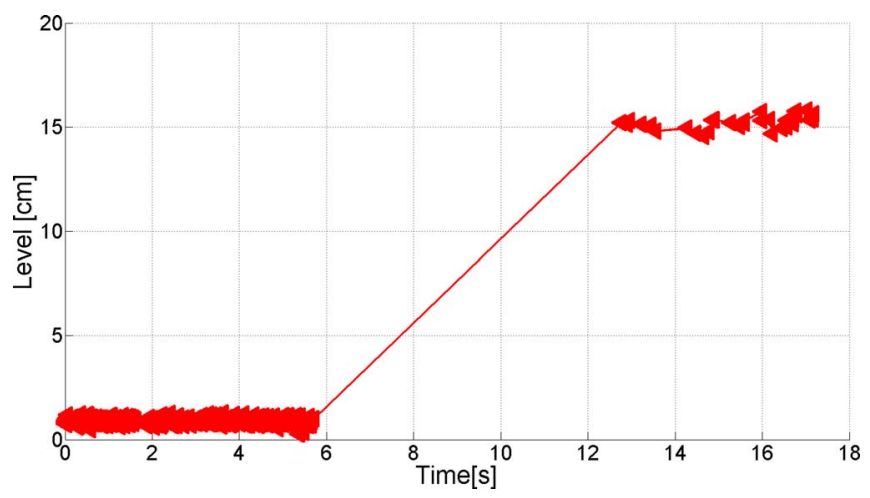

FIG. 14. Liquid level measurements during an industrial filling process. Focus before the target (see Fig. 6(a)). Average distance of the surface was around $40 \mathrm{~cm}$.

\section{CONCLUSION}

This contribution presents the development of a compact and low-cost self-mixing liquid level measurement instrument, able to measure directly the position of the free surface of the water. A dedicated electronics based on FPGA device has been developed for high-speed acquisition, needed for this particular application. In addition, an algorithm for signal analysis and valid data selection has been proposed and tested. Method and optical configuration have been studied in order to overcome the problem of water movement and surface random reflections. It has been proven that a water surface rippled at high-frequency has an optical behavior similar to a diffusive surface, and it can be measured by focusing the laser beam shortly after the free surface. On the contrary, a still water can be measured through the optical configuration shown in Fig. 6(a). The instrument ability to measure with both a flat and a rippled surface has been demonstrated by monitoring an industrial filling process with a filling velocity of about $2.5 \mathrm{~cm} / \mathrm{s}$. Compact size and low cost are additional features of this instrument composed only by a small electronic circuit and a FPGA board. Future developments concern the possibility of implementing an auto-focus system, with very high speed, in order to follow the sudden changes in the reflected signal.

${ }^{1}$ H.-H. Hao and J.-Q. Xiong, ICCASM 2010 - 2010 International Conference on Computer Application and System Modeling 11, V11682-V11684 (2010).

${ }^{2}$ J. C. Chun, T. S. Kim, J. M. Kim, Z. S. Lim, and W. S. Park, IEEE MTT-S International Microwave Symposium Digest 1, 2251-2254 (2001).

${ }^{3}$ J. E. Antonio-Lopez, J. J. Sanchez-Mondragon, P. LiKamWa, and D. A. May-Arrioja, Opt. Lett. 36(17), 3425 (2011).

${ }^{4}$ I. K. Ilev and R. W. Waynant, Rev. Sci. Instrum. 70(5), 2551 (1999).

${ }^{5}$ S. F. Jacobs and J. G. Small, Appl. Opt. 20(20), 3508 (1981).

${ }^{6}$ G. Giuliani, M. Norgia, S. Donati, and T. Bosch, J. Opt. A: Pure Appl. Opt. 4(6), S283 (2002).

${ }^{7}$ S. Donati, Laser Photonics Rev. 6, 393 (2012).

${ }^{8}$ M. Norgia and S. Donati, IEEE Trans. Instrum. Meas. 52(6), 1765 (2003).

${ }^{9}$ C. Bes, G. Plantier, and T. Bosch, IEEE Trans. Instrum. Meas. 55(4), 1101 (2006).

${ }^{10}$ A. Magnani and M. Norgia, IEEE J. Quantum Electron. 49(9), 765 (2013).

${ }^{11}$ L. Scalise, Y. Yu, G. Giuliani, G. Plantier, and T. Bosch, IEEE Trans. Instrum. Meas. 53(1), 223 (2004).

${ }^{12}$ F. Gouaux, N. Servagent, and T. Bosch, Appl. Opt. 37(28), 6684 (1998).

${ }^{13}$ M. Norgia, A. Magnani, and A. Pesatori, Rev. Sci. Instrum. 83(4), 045113 (2012).

${ }^{14}$ M. Norgia, A. Pesatori, and L. Rovati, IEEE Trans. Instrum. Meas. 59(5), 1233 (2010).

${ }^{15}$ L. Campagnolo, M. Nikolic, J. Perchoux, Y. L. Lim, K. Bertling, K. Loubière, L. Prat, A. D. Rakic, and T. Bosch, Microfluid. Nanofluid. 14, 113 (2013).

${ }^{16}$ S. K. Özdemir, S. Takamiya, S. Ito, S. Shinohara, and H. Yoshida, IEEE Trans. Instrum. Meas. 49(5), 1029 (2000).

${ }^{17}$ U. Zabit, F. Bony, T. Bosch, and A. D. Rakic, IEEE Photonics Technol. Lett. 22(6), 410 (2010).

${ }^{18}$ S. Donati, M. Norgia, and G. Giuliani, Appl. Opt. 45(28), 7264 (2006).

${ }^{19}$ A. Magnani, A. Pesatori, and M. Norgia, Appl. Opt. 51(21), 5318 (2012).

${ }^{20}$ S. Donati and M. Norgia, IEEE J. Sel. Top. Quantum Electron. 20(2), 6900108 (2014).

${ }^{21}$ R. Lang and K. Kobayashi, IEEE J. Quantum Electron. 16, 347 (1980).

${ }^{22}$ M. Norgia, A. Pesatori, M. Tanelli, and M. Lovera, IEEE Trans. Instrum. Meas. 59, 1368 (2010).

${ }^{23}$ K. Petermann, IEEE J. Sel. Top. Quantum Electron. 1(2), 480 (1995).

${ }^{24}$ G. Giuliani and M. Norgia, IEEE Photonics Technol. Lett. 12(8), 1028 (2000). 